\title{
Demonstration of 2-Hydroxybenzoylglycine As a Drug Binding Inhibitor in Newborn Infants
}

\author{
Byungse Suh, Sandra J. Wadsworth, and Diane M. Lichtenwalner \\ Section of Infectious Diseases, Department of Medicine, Temple University Health Sciences Center, Philadelphia, Pennsylvania 19140
}

\begin{abstract}
Newborn infants have drug binding defects that share similarities to those of uremic subjects. Since 2-hydroxybenzoylglycine has been chemically defined to be a major drug binding inhibitor in uremia, a search for the presence of a similar compound in the sera of newborn infants was made.

An organic substance that has the characteristics of 2-hydroxybenzoylglycine as supported by the retardation factor values on thin-layer chromatograms, retention times of high performance liquid chromatograms, fluorescence emission spectra, and mass spectrum has been demonstrated to be present in the majority of the neonatal sera studied.

A strong positive correlation between the levels of the binding inhibitor and the extent of binding defects for nafcillin has been observed. The substance could effectively reduce the total bilirubin concentration when added to the cord sera specimens. It is concluded that 2-hydroxybenzoylglycine plays an important role in drug binding defects observed in the newborn, and the inhibitor may also play a part in the precipitation of bilirubin-induced neurotoxicity in neonates when the substance is abnormally elevated.
\end{abstract}

\section{Introduction}

Plasma protein binding of drugs is a reversible and rather nonselective interaction between a small drug molecule and a biological macromolecule, plasma protein. The binding process is very sensitive to many surrounding factors and can therefore be altered by the changes in such parameters, which may include drug and protein concentration, temperature, $\mathrm{pH}$, the nature of the buffer used, and the presence of endogenous substances. The presence of a number of pathophysiological conditions has been associated with decreased protein binding of many drugs. Patients with severe renal failure and the newborn are two well-known groups with such binding defects (1-5).

Newborn infants have drug binding defects that share some similarities to those of uremic subjects in that both groups have reduced binding of many acidic drugs and the binding defects can be corrected by activated charcoal treatment at an acidic $\mathrm{pH}(3,5)$. Recently 2-hydroxybenzoylglycine has been chemically defined to be a major drug binding inhibitor in uremia, although the steps involved in the genesis of the sub-

Address correspondence to Dr. Suh.

Received for publication 16 June 1986 and in revised form 23 March 1987.

J. Clin. Invest.

(c) The American Society for Clinical Investigation, Inc.

$0021-9738 / 87 / 10 / 1125 / 07 \$ 2.00$

Volume 80, October 1987, 1125-1131 stance have not been elucidated (6). This study was undertaken to search for the presence of a similar substance in sera of neonates. This paper reports the findings that confirm the presence of 2-hydroxybenzoylglycine in elevated levels in the majority of neonatal sera studied. Its potential role in the newborn as a drug binding inhibitor and as a potent bilirubin displacing agent is discussed.

\section{Methods}

Chemicals. Chemicals were purchased from the following sources: 2-hydroxybenzoic acid from Fisher Scientific Co. (Pittsburgh, PA); dicyclohexylcarbodiimide from Eastman Kodak Co. (Rochester, NY); p-dioxane from Aldrich Chemical Co. (Milwaukee, WI); 5,5-diphenylhydantoin, $N$-hydroxysuccinamide, and 2-hydroxybenzoylglycine from Sigma Chemical Co. (St. Louis, MO); $n$-butyl chloride (glass distilled) from Burdick \& Jackson Chemical Co. (Muskegon, MI); methylene chloride from Fisher Scientific Co.; and $\left[{ }^{14} \mathrm{C}\right] \mathrm{glycine}$ from New England Nuclear (Boston, MA). Nafcillin sodium was supplied by Wyeth Laboratories (West Chester, PA).

Serum samples. Normal healthy volunteers were used to obtain either pooled or individual serum specimens to be used as controls. The umbilical cord serum specimens were obtained from the Immunology Laboratory of Temple University Hospital; only the serum specimens with nonreactive syphilis serology were used. The paired maternal and cord blood specimens were obtained at the time of delivery. All blood specimens were obtained with informed consent. Serum specimens were kept frozen at $-20^{\circ} \mathrm{C}$ until use.

Protein binding measurements. Equilibrium dialysis as described previously $(7,8)$ was used to determine the degree of protein binding. The final concentration of nafcillin was $20 \mu \mathrm{g} / \mathrm{ml}$. The dialysis was performed at $4^{\circ} \mathrm{C}$ with $\mathrm{pH}$ adjusted to 7.4 .

Preparation of the extract with drug binding inhibitor from cord serum. Normal pooled human adult and cord sera were extracted with methylene chloride similar to the method previously described (9). The organic solvent extract prepared from a 5-ml serum sample was evaporated to $\sim 0.2-0.3 \mathrm{ml}$ and used for thin-layer chromatography.

Thin-layer chromatography of extracts. Aliquots of normal adult or cord serum extracts as described above were applied to silica gel 60 plates (E. Merck, Cherry Hill, NJ) and developed by ascending chromatography by the method previously described (6). Visualization of the potential binding inhibitor was accomplished using an ultraviolet (UV) light lamp as previously reported (6).

Chemical synthesis of $\left[{ }^{14} \mathrm{C}\right] 2$-hydroxybenzoylglycine. A modification of the active ester method $(10)$ was used to synthesize ${ }^{14} \mathrm{C}$-labeled 2-hydroxybenzoylglycine. The carbon atom of the carboxyl group of the glycine moiety was isotope labeled. The purity of the final product was tested by thin-layer chromatography as described above.

Identification of binding inhibitor by mass spectrometry. An area of the thin-layer chromatogram corresponding to the location of the binding inhibitor was scraped from the plate, eluted with methanol, and the extract was filtered through sintered glass. The methanol was evaporated to dryness, and $0.1 \mathrm{ml} \mathrm{N}$-O-bis(trimethylsilyl)trifluoracetamide with $1 \%$ trimethylchlorsilane (Supelco, Inc., Bellefonte, PA) was added to the residue. The vial was taken for analysis. A quadrupole mass spectrometer (model 1050; Finnigan Corp., Sunnyvale, CA) scanning from 40 to 400 atomic mass units in $1 \mathrm{~s}$ in the electron impact 
mode $(70 \mathrm{eV})$ was used. Sample introduction was through a 2-m $\times 2$-mm Dexsil 300 column, programmed from $150^{\circ}$ to $375^{\circ} \mathrm{C}$ at $30^{\circ} \mathrm{C} / \mathrm{min}$. The helium carrier $\left(30 \mathrm{~cm}^{3} / \mathrm{min}\right)$ was removed via a jet separator, and the enriched sample introduced into the mass spectrometer ion source.

Quantitation of 2-hydroxybenzoylglycine by high performance liquid chromatography. A quantitative method for determination of 2-hydroxybenzoylglycine in serum or buffer was developed using high performance liquid chromatography.

The chromatographic equipment was constructed from commercially available components. A Minipump sample delivery system, 6000 per square inch on the gauge (psig) ${ }^{1}$ (Laboratory Data Control, Riviera Beach, FL), and a UV/visible optical unit, type 6 (ISCO Inc., Lincoln, NE) were used with a universal sample injector, 7,000 psig

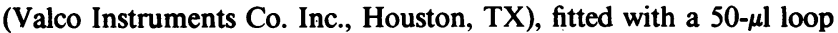
(Alltech Assoc., Deerfield, IL). A reverse-phase column (Microsorb C-18; Rainin Instrument Co., Inc., Ridgefield, NJ) was used. A UA-5 absorbance/fluorescence detector (ISCO Inc.) was used to record the UV absorbance.

A solvent system consisting of acetic acid/water/methanol (41:779:220, vol/vol/vol) was used with $2,100 \mathrm{psi}$ pressure being applied. The flow rate was at $1.4 \mathrm{ml} / \mathrm{min}$. Detection of 2-hydroxybenzoylglycine was made at $310 \mathrm{~nm}$. The sample preparation was accomplished by an organic solvent extraction method. To 2.0-ml aliquots of serum or buffer specimens containing 2-hydroxybenzoylglycine was added $0.4 \mathrm{ml} 5 \mathrm{~N}-\mathrm{HCl}$ followed by addition of $7.5 \mathrm{ml}$ methylene chloride. After a brief mixing in a Vortex mixer, the specimens were centrifuged for $5 \mathrm{~min}$ in a table top clinical centrifuge. The bottom solvent layer was removed into another centrifuge tube, the top aqueous layer was subjected to second extraction, and the organic layers were pooled. The pooled solvent layer was brought to dryness using a low temperature block heater (model 2090; Lab-Line Instruments Inc., Melrose Park, IL). The specimen was reconstituted by dissolving it in $60 \mu \mathrm{l}$ of the mobile phase solvent and the equal volume of internal standard ( $O$-anisic acid in acetonitrile, $4 \mathrm{mg} / \mathrm{dl}$ ). $50 \mu \mathrm{l}$ of the sample were introduced into the sample injector for quantitation.

A standard curve was generated by plotting the ratio of the height of the 2-hydroxybenzoylglycine peak to that of the internal standard peak against the established standard concentrations of the standard. Leastsquare linear regression analysis was used to generate the curve and calculate concentrations in the control and serum samples.

Assay procedures. Serum total protein and albumin concentrations were determined by the methods of Gornall et al. (11) and Doumas et al. (12), respectively. Free fatty acid levels and bilirubin concentration were estimated by the procedures of Laurell and Tibbling (13) and Roth (14), respectively. These assay procedures were done in duplicate, and optical absorbance was measured in a spectrophotometer (Spectronic 21; Bausch \& Lomb Inc., Rochester, NY). Fluorometric assay for nafcillin (15) was used. Radioactivity was measured in a small aliquot of serum or buffer $(50 \mu \mathrm{l})$ in $3 \mathrm{ml}$ of Aquasol scintillation fluid (New England Nuclear). A refrigeratable scintillation counter (model LS-6800; Beckman Instruments, Inc., Irvine, CA) was used.

\section{Results}

Protein binding of nafcillin in cord serum. Protein binding values were measured by equilibrium dialysis procedure as described in Methods. The binding measurements were made in both normal adult and cord serum specimens for comparison. The results obtained are summarized in Table I. The binding values of nafcillin in cord serum specimens are significantly lower than those of normal adult serum samples.

Effect of $n$-butyl chloride extraction on protein binding. Both normal adult and cord serum specimens were extracted

1. Abbreviation used in this paper: psig, per square inch on the gauge.
Table I. Nafcillin Binding in Neonates Vs. Normal Adults

Nafcillin binding

Normal pooled human serum

$85.20 \pm 0.34$

Neonatal serum

$75.02 \pm 1.71^{\ddagger}$

Neonatal serum after extraction with $n$-butyl

chloride (pH 3.0)

$83.57 \pm 1.34^{\S}$

Neonatal serum dialyzed against human

serum albumin (48 h)

$82.36 \pm 0.78^{\S}$

* Values are means \pm SD and are derived from six different sera per value.

$¥$ Values are statistically lower than normal adult values with $P<0.005$.

$\$$ Values are statistically greater than pretreatment values with

$P<0.005$.

with $n$-butyl chloride as previously described (9) and the results shown in Table I were obtained. It has been well demonstrated that the organic solvent extraction causes a significant improvement in binding of nafcillin in cord specimens, and the binding values are comparable with those of normal adult serum specimens.

Thin-layer chromatography of methylene chloride extract. While both $n$-butyl chloride and methylene chloride are capable of extracting 2-hydroxybenzoylglycine from serum specimens, methylene chloride was shown to be the superior solvent for the extraction purpose. In various serum specimens, including normal adult human serum, the identical specimen, spiked with authentic 2-hydroxybenzoylglycine and cord serum specimens were extracted with methylene chloride at an acidic $\mathrm{pH}$ at room temperature. The organic solvent layers were used to detect the presence of 2-hydroxybenzoylglycine. The organic solvent layers were brought to dryness, the residue was redissolved in $0.2 \mathrm{ml}$ methanol, and the alcohol solution was used to perform thin-layer chromatography. The presence of 2-hydroxybenzoylglycine was demonstrated using a short wave $(254 \mathrm{~nm})$ UV light. The results shown in Fig. 1 were obtained. The results demonstrated that a band that has a similar retardation factor $\left(R_{\mathrm{f}}\right)$ value to that of 2 -hydroxybenzoylglycine is present in cord serum specimen. Of the cord specimens tested for the presence of 2-hydroxybenzoylglycine,

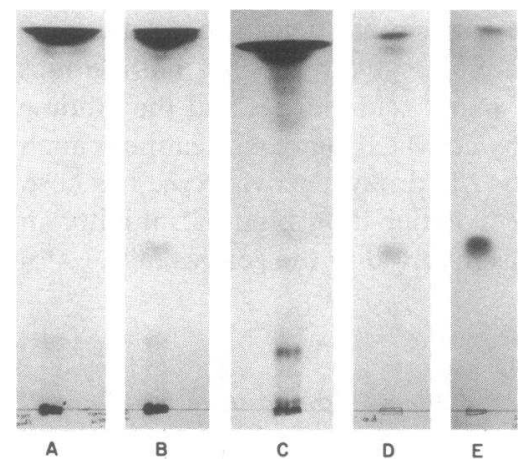

Figure 1. Thin-layer chromatograms of serum extracts. Lane $A$, extract of normal adult serum; lane $B$, extract of normal adult serum spiked with 2-hydroxybenzoylglycine; lane $C$ extract of cord serum; lane $D$, purified fraction containing the drug binding inhibitor in cord serum from lane $C$; and lane $E$, pure 2hydroxybenzoylglycine. Solvent system was petroleum ether/ether/ absolute ethyl alcohol/glacial acetic $\operatorname{acid}(24: 6: 7: 2, \mathrm{vol} / \mathrm{vol} / \mathrm{vol} / \mathrm{vol})$ and detection was accomplished by visualization with shortwave (254 nm) UV light. 
$>92 \%$ revealed the presence of such binding inhibitor, while none of the normal adult serum specimens (50 volunteers) tested showed similar results.

Seven to nine spots corresponding to that of known 2-hydroxybenzoylglycine in thin-layer chromatography plates were scraped and pooled followed by an extraction with a small amount of methanol. When the methanol solution was subjected to a fluorescence spectrometry, the emission spectra shown in Fig. 2 were obtained. It is identical to that of 2-hydroxybenzoylglycine.

Mass spectrum of binding inhibitor. The mass spectrum of authentic bis-trimethylsilyl $N$-(2-hydroxybenzoyl) glycine is shown in Fig. $3 \mathrm{~A}$. A weak molecular ion at $\mathrm{m} / \mathrm{z} 339$, loss of methyl (m/z 324), benzoyl cleavage $(\mathrm{m} / \mathrm{z} 193)$, and $\mathrm{m} / \mathrm{z} 206$ are prominent features of the spectrum. Fig. $3 B$ shows the mass spectrum of the major gas chromatographic peak. The retention times of authentic sample and unknown were identical (3.9 min). It contains all of the key ions found in bis-trimethylsilyl $N$-(2-hydroxybenzoyl) glycine. It also has a prominent $\mathrm{m} / \mathrm{z}$ 100; however, ion chromatograms of $\mathrm{m} / \mathrm{z} 100 \mathrm{vs}$. 193, 206, and 339 clearly show that two chromatographic peaks are present which are incompletely resolved. A computer-assisted search routine based on a 42222 index library found the greatest similarity of fit between the unknown and bis-trimethylsilyl $N$-(2-hydroxybenzoyl) glycine.

Quantitation of 2-hydroxybenzoylglycine. A high performance liquid chromatography was used to develop a method to quantitate the levels of 2-hydroxybenzoylglycine. The procedure is described in detail in the Methods section. Typical chromatograms of the internal standard and 2-hydroxybenzoylglycine extracted from serum are shown Fig. 4 . The retention times for the internal standard and 2-hydroxybenzoylglycine were 10.3 and $7.9 \mathrm{~min}$, respectively. A standard curve generated according to the method above is shown in Fig. 5. Extraction efficiency was investigated using $\left[{ }^{14} \mathrm{C}\right] 2$ hydroxybenzoylglycine in concentration ranges from 0 to 5.5 $\mu \mathrm{M}$. The recovery rate was $49-52 \%$. The correlation coefficients for the high performance liquid chromatography standard curves ranged from 0.997 to 1.000 .

The stability of 2-hydroxybenzoylglycine to various temperatures was studied by subjecting serum specimens containing the binding inhibitor to freezing, thawing, and incubation at room temperature for several hours. These conditions showed little effect on the levels of 2-hydroxybenzoylglycine.

The cord serum extracts, 33 of 39 specimens tested, revealed the presence of a substance that has a similar $R_{\mathrm{f}}(0.43)$ value to that of 2-hydroxybenzoylglycine, while none of over 50 normal specimens showed similar findings. When quanti-

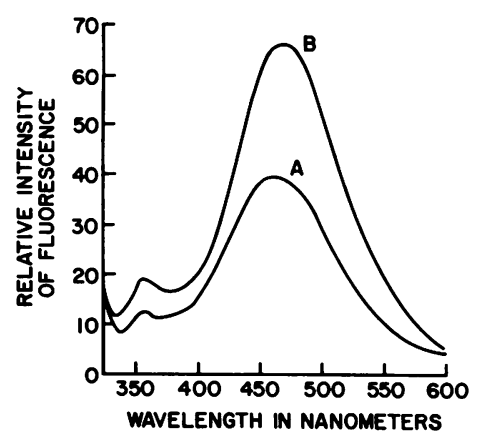

Figure 2. Fluorescence emission spectra of the drug binding inhibitor from cord serum. The spectra were measured in methanol. Excitation was at $305 \mathrm{~nm}$. Curve A, purified drug binding inhibitor; curve B, authentic 2-hydroxybenzoylglycine. tated by the high performance liquid chromatography, the levels of 2-hydroxybenzoylglycine in the cord serum specimens ranged from 0 to $6.15 \mu \mathrm{M}$, while the levels in maternal serum specimens ranged from 0 to $3.08 \mu \mathrm{M}$. When paired maternal and cord serum specimens were studied for more direct comparison, the levels of the binding inhibitor were demonstrated to be higher in the cord specimens in the majority of the cases $(P<0.05)$. These results are summarized in Table II.

Correlation between the levels of 2-hydroxybenzoylglycine and nafcillin binding. To investigate the effect of 2-hydroxybenzoylglycine on serum protein binding of nafcillin, the correlation between the levels of 2-hydroxybenzoylglycine and the extent of binding defects of nafcillin was studied. The levels of 2-hydroxybenzoylglycine were measured by the high performance liquid chromatography as described above and protein binding of nafcillin was estimated by equilibrium dialysis. The binding studies were performed in triplicate in cord serum and normal adult serum specimens as well. The results are presented in Fig. 6. There is a strong positive correlation between the levels of 2-hydroxybenzoylglycine and the extent of binding defect of nafcillin. Addition of increasing amounts of 2-hydroxybenzoylglycine to normal adult sera caused a concomitant reduced binding of nafcillin. The extent of binding defects induced closely paralleled the values observed in neonatal sera when the levels of 2-hydroxybenzoylglycine were taken into consideration.

Levels of albumin, free fatty acids, and bilirubin. The concentration ranges for albumin, free fatty acids, and bilirubin in the cord serum specimens studied were 3.05 to $4.0 \mathrm{~g} / \mathrm{dl}, 425$ to $535 \mu \mathrm{eq} /$ liter, and 1.8 to $8.6 \mathrm{mg} / \mathrm{dl}$, respectively. The methods used were as those described in the Methods section.

Effect of 2-hydroxybenzoylglycine on total bilirubin concentration in cord sera. A batch of pooled cord serum was used as the source of serum containing elevated levels of total serum bilirubin. The effects of several chemical agents including salicylate, aspirin, sulfisoxazole, nafcillin, and 2-hydroxybenzoylglycine on the levels of total bilirubin were investigated by adding the agents to serum followed by centrifugation in semipermeable cones. The $\mathrm{pH}$ of serum pool containing potential bilirubin displacing agent was adjusted to 7.4 and $0.5-\mathrm{ml}$ aliquots were used for centrifugation for $5 \mathrm{~min}$. The procedure used was basically that of Singhvi et al. (16). Membrane ultrafiltration cones (CF50A CentriFlo; Amicon Corp., Lexington, MA) were used. The total bilirubin levels were measured before and after the centrifugation. The controls were cord serum specimens treated with same way without the displacing agent. The difference in total bilirubin levels before and after the centrifugation was used as the measure of bilirubin displaced. The results are summarized in Table III. The binding inhibitor, 2-hydroxybenzoylglycine was shown to be the most effective displacing agent among the agents tested.

\section{Discussion}

It has been well documented that the newborn has a decreased plasma protein binding for many drugs $(4,5,17)$. Such defective drug binding appears to be due not only to the reduced protein concentration at the time of birth but also to the presence of several other factors that have been proposed as the possible explanations. These include $(a)$ a qualitative differ- 

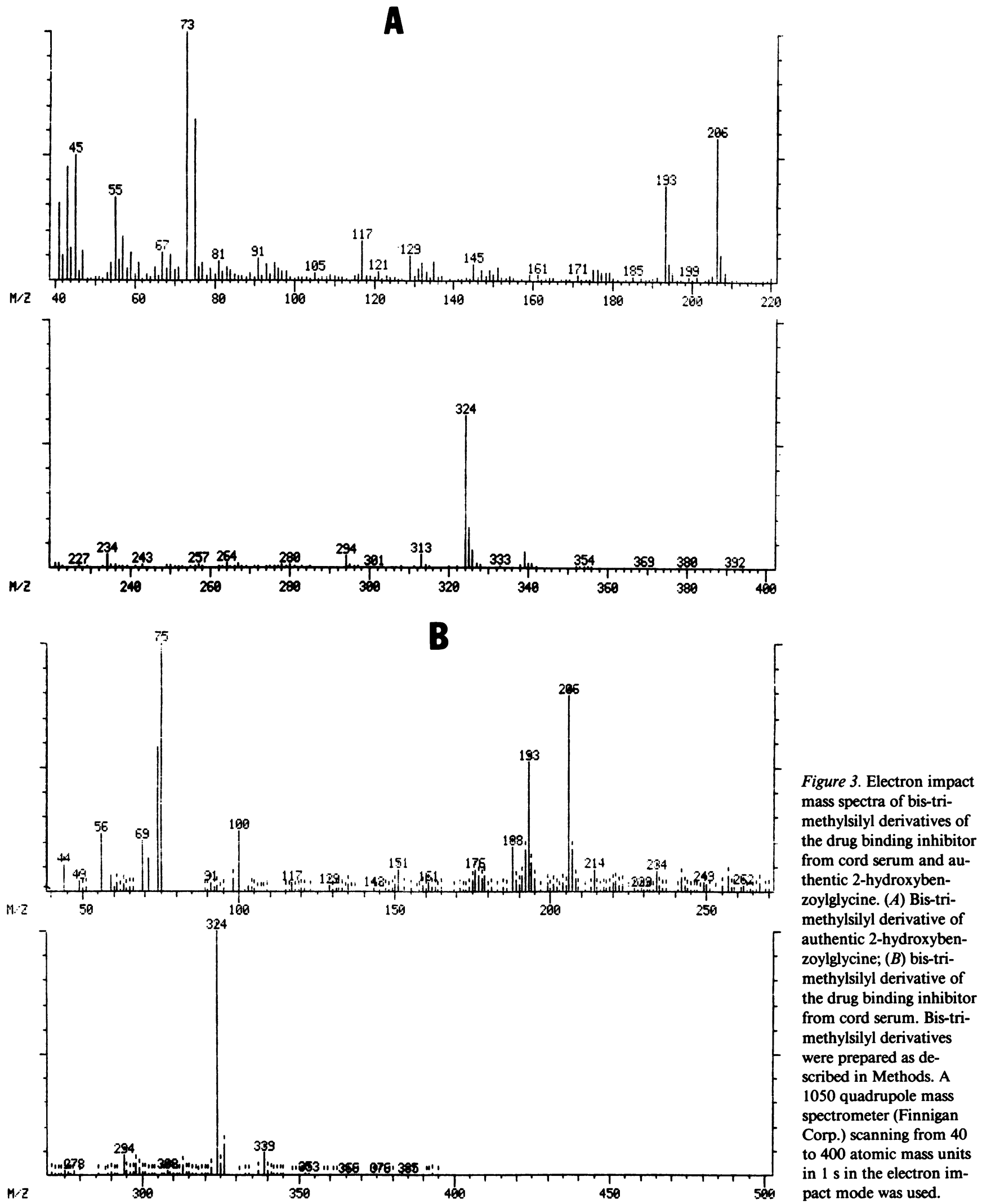

ence in albumin structure $(18,19),(b)$ high concentrations of endogenous metabolic products such as bilirubin and free fatty acids (20-22), (c) presence of other unknown endogenous binding inhibitor(s) which may compete with pharmacological agents for protein binding (5), and $(d)$ changes in other physiological factors such as $\mathrm{pH}(23)$.

The protein binding defects seen in the newborn share several similarities to those of uremic patients in that neonates 


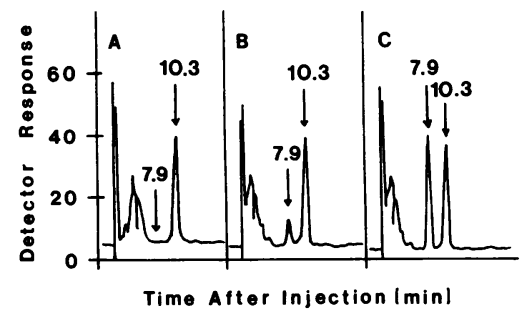

zoylglycine. See Methods for experimental conditions.

have reduced binding for many acidic drugs including phenytoin, sulfonamides, barbiturates, salicylates, and penicillins (1-5), and the defects can be corrected by the activated charcoal treatment at acidic $\mathrm{pH}(3,5)$. In uremia 2-hydroxybenzoylglycine has been shown to be a major drug binding inhibitor and it has been demonstrated to be elevated in a majority of the patients with severe renal failure $(6,24)$. Therefore a search for the presence of a similar compound in the newborn was undertaken. Nafcillin was chosen as the test agent to measure the degree of protein binding. We have an accurate assay method for nafcillin and it represents a highly bound acidic drug for which the newborn has reduced drug binding (17).

As well demonstrated in Table I, neonates have significantly lower binding values for nafcillin as compared with those of normal adult volunteers. Furthermore, it was shown that the binding defects could be corrected by $n$-butyl chloride extraction at an acidic $\mathrm{pH}$. This clearly indicated that the organic solvent most likely contained the drug binding inhibitor(s). The analytical methods that were successfully used in the identification of 2-hydroxybenzoylglycine from uremic sera were again used. These included thin-layer chromatography, UV absorption spectrometry, fluorescence emission spectroscopy, high performance liquid chromatography, and mass spectrometry. The methylene chloride extracts of cord serum specimens revealed the presence of a fluorescent compound that had a similar $R_{\mathrm{f}}$ value to that of commercially available 2-hydroxybenzoylglycine. The spots containing the fluorescent material were scraped and pooled followed by extraction with methanol for further identification. When analyzed by fluorescence emission spectrometry, the compound had the spectrum which was identical to that of pure 2-hydroxybenzoylglycine. The compound was shown to possess the same retention time on high performance liquid chromatography as the standard 2-hydroxybenzoylglycine. When the unknown binding inhibitor was subjected to mass spectrometry, the compound was shown to contain all the key ions found in the authentic 2-hydroxybenzoylglycine, conclusively confirming the unknown compound to be 2-hydroxybenzoylglycine.

To investigate the role of this binding inhibitor an attempt

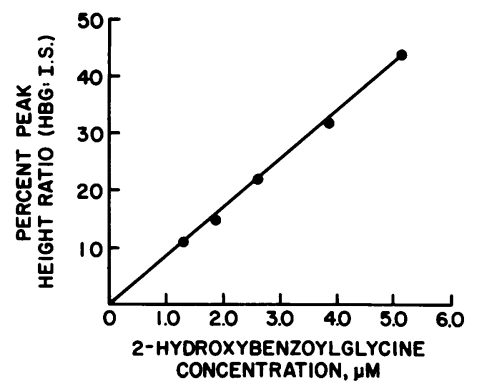

Figure 5. High performance liquid chromatography standard curve for 2hydroxybenzoylglycine (HBG). Correlation coefficient, 0.997; internal standard (I.S.), $o$-anisic acid. See Methods for detailed experimental conditions.
Table II. Comparison of the Levels of 2-Hydroxybenzoylglycine in Paired Maternal and Cord Serum Specimens*

\begin{tabular}{rll}
\hline & \multicolumn{2}{l}{ Levels of 2-hydroxybenzoylglycine } \\
\cline { 2 - 3 } Specimen no. & Maternal $^{\ddagger}$ & Cord $^{5}$ \\
\hline & $\mu M$ & $\mu M$ \\
1 & 3.08 & 3.85 \\
2 & 0 & 1.54 \\
3 & 3.08 & 3.08 \\
4 & 0 & 1.54 \\
5 & 0 & 0 \\
6 & 0 & 1.54 \\
7 & 0 & 1.54 \\
8 & 0 & 2.31 \\
9 & 0 & 0 \\
10 & 1.79 & 6.15 \\
11 & 3.08 & 0 \\
12 & 0 & 2.31 \\
& &
\end{tabular}

* The blood specimens were obtained at the time of delivery.

${ }^{\ddagger}$ The specimens were obtained from mothers who were not on salicylate therapy.

${ }^{\S}$ The specimens were obtained from full-term infants only.

was made to correlate the levels of 2-hydroxybenzoylglycine as assayed by the high performance liquid chromatography procedure to the extent of binding defects for nafcillin. A strong positive correlation was demonstrated between the two parameters. These results could be reproduced by adding 2-hydroxybenzoylglycine to normal pooled adult serum to the levels seen in cord sera. Therefore it appears apparent that 2-hydroxybenzoylglycine is present in high concentrations in the neonatal sera and it plays a role of drug binding inhibitor, although other roles that the compound may play in the newborn are unclear.

Since the drug binding inhibitor was capable of displacing nafcillin, a highly bound acidic drug, its ability to displace bilirubin from neonatal serum protein was investigated. Other pharmacological agents that are known to be capable of displacing bilirubin were also studied for comparison. These included a sulfonamide, salicylate, aspirin, and nafcillin. The binding inhibitor was demonstrated to be the most effective

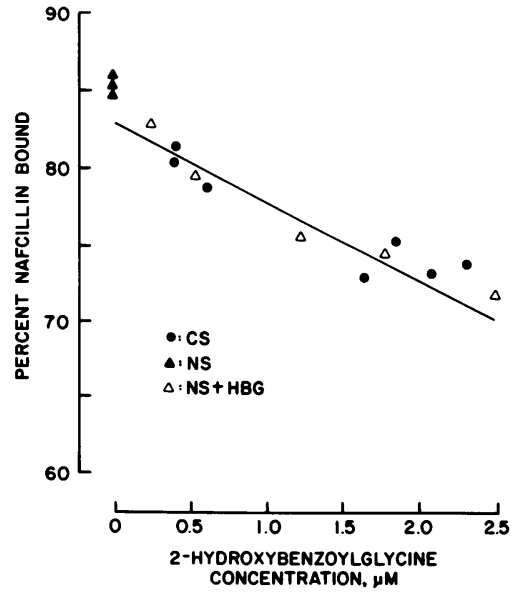

Figure 6. Correlation between the levels of 2 hydroxybenzoylglycine (HBG) and the extent of drug binding defects for nafcillin. Each point represents nafcillin binding values in serum. ( $\Delta$ ) Normal adult serum (NS); $(\Delta)$ normal adult serum with addition of 2-hydroxybenzoylglycine in concentrations indicated (NS + HBG); (•) cord serum (CS). 
Table III. Effect of 2-Hydroxybenzoylglycine on Total Bilirubin Concentration in Cord Serum*

\begin{tabular}{ll}
\hline $\begin{array}{l}\text { Bilirubin displacing agents, } \\
\text { final concentration }(5.0 \mu \mathrm{M})\end{array}$ & $\begin{array}{l}\text { Total bilirubin } \\
\text { concentration }\end{array}$ \\
& $m g / d l$ \\
None & $3.44 \pm 0.16^{\ddagger}$ \\
2-Hydroxybenzoic acid & $3.20 \pm 0.12$ \\
Aspirin & $2.96 \pm 0.16$ \\
Sulfisoxazole & $2.72 \pm 0.20$ \\
Nafcillin & $2.24 \pm 0.20$ \\
2-Hydroxybenzoylglycine & $0.49 \pm 0.17$ \\
\end{tabular}

* A batch of pooled cord serum with total bilirubin concentration of $3.44 \mathrm{mg} / \mathrm{dl}$ was used. $\mathrm{pH}$ of the serum was adjusted to $7.40 \mathrm{after}$ the addition of bilirubin displacing agents. The specimens were centrifuged in semipermeable cones for $5 \mathrm{~min}$.

* The values represent means of three separate determinations 土standard deviation.

bilirubin displacing agent among those tested. The levels of bilirubin displacing agents studied were $0-5 \mu \mathrm{M}$. It is probable that 2-hydroxybenzoylglycine may play an important role in the genesis of bilirubin-induced neurotoxicity in the newborn (i.e., kernicterus).

The precise biochemical mechanisms involved in the production of 2-hydroxybenzoylglycine in the newborn are unclear. Chemically, 2-hydroxybenzoylglycine is a glycine conjugate of salicylic acid, and it has previously been demonstrated that human neonates excreted considerably more glycine conjugates of salicylates than did the other salicylate derivatives such as unmetabolized salicylic acid, salicyl phenolic glucuronide, and gentisic acid. In addition, the other known metabolite of salicylate, salicyl acyl glucuronide, could not be detected in the same population (25). Therefore, it appears quite evident that the newborn handles salicylates by the preferential glycine conjugation process. The final predominant product, 2-hydroxybenzoylglycine, will tend to accumulate in the newborn due to the capacity-limited elimination and consequential prolonged half-life in this age group. At birth, renal function is greatly reduced if compared with children and adult values (26). It is understandable that compounds that depend upon renal function for their excretion would be disposed of at a very slow rate in the newborn and tend to accumulate, and 2-hydroxybenzoylglycine is a good example.

To investigate the origin of 2-hydroxybenzoylglycine in the cord serum specimens, paired maternal and cord sera obtained at the time of delivery were analyzed for the levels of 2-hydroxybenzoylglycine. In almost every paired case, the level of the binding inhibitor in the cord serum specimen was significantly higher than that in the maternal specimen. This finding strongly suggests that the origin of 2-hydroxybenzoylglycine is most likely fetal rather than maternal. However, the source of the substrate, salicylate, could well be maternal in origin since exogenous sources of salicylates are multiple. They include some obvious sources such as salicylate containing medications, but more importantly various dietary substances including fruits and vegetables (27), cow's milk (28), and food additives (29) are known to contain substantial levels of salicylates.

In summary, it has been demonstrated that 2-hydroxybenzoylglycine is present at elevated levels in cord serum specimens, and it is proposed that 2-hydroxybenzoylglycine plays an important role in drug binding defects observed in neonates. The precise mechanisms involved in the genesis of the binding inhibitor in the newborn are not well understood and will require further investigation.

\section{Acknowledgments}

Mass spectrometry was performed at National Medical Services, Willow Grove, PA. We wish to thank Dr. Mark R. Lichtenwalner for performing the procedure and for his interpretation of the spectrum. We also wish to thank Dr. Fredric Rieders for making the mass spectrometer available to us. The authors are also indebted to Dr. Martin Farber and staff members of the Delivery Room in the Department of Obstetrics and Gynecology at Albert Eistein Medical Center (Northern Division), Philadelphia, PA., for their assistance in organization and collection of the paired maternal and cord blood specimens.

This study was supported in part by grant 5R01 AM26807 (to Dr. Suh) from the National Institute of Arthritis, Diabetes, Digestive and Kidney Diseases.

\section{References}

1. Reidenberg, M. M., I. Odar-Cederlöf, C. von Bahr, O. Borgå, and F. Sjöqvist. 1971. Protein binding of diphenylhydantoin and desmethylimipramine. N. Engl. J. Med. 285:264-267.

2. Reidenberg, M. M., and M. Affrime. 1973. Influence of disease on binding of drugs to plasma proteins. Ann. NY Acad. Sci. 226:115126.

3. Craig, W. A., M. A. Evenson, K. P. Sarver, and J. P. Wagnild. 1976. Correction of protein binding defect in uremic sera by charcoal treatment. J. Lab. Clin. Med. 87:637-647.

4. Kurz, H., A. Mauser-Ganshown, and H. H. Stickel. 1977. Differences in the binding of drugs to plasma proteins from newborn and adult man. I. Eur. J. Clin. Pharmacol. 11:463-467.

5. Chignell, C. F., E. S. Vesell, D. K. Starkweather, and C. M. Berlin. 1971. The binding of sulfaphenazole to fetal, neonatal, and adult human plasma albumin. Clin. Pharmacol. Ther. 12:897-901.

6. Lichtenwalner, D. M., B. Suh, and M. R. Lichtenwalner. 1983. Isolation and chemical characterization of 2-hydroxybenzoylglycine as a drug binding inhibitor in uremia. J. Clin. Invest. 71:1289-1296.

7. Kunin, C. M. 1966. Clinical pharmacology of the new penicillins. I. The importance of serum protein binding in determining antimicrobial activity and concentration in serum. Clin. Pharmacol. Ther. 7:166-188.

8. Craig, W. A., and B. Suh. 1986. Protein binding and the antimicrobial effects: methods for the determination of protein binding. In Antibiotics in Laboratory Medicine. V. Lorian, editor. Williams \& Wilkins, Baltimore. 477-514.

9. Lichtenwalner, D. M., B. Suh, B. Lorber, M. R. Rudnick, and W. A. Craig. 1981. Partial purification and characterization of the drug-binding-defect inducer in uremia. J. Lab. Clin. Med. 97:72-81.

10. Van Brussel, W., and C. F. Van Sumere. 1978. N-Acylamino acids and peptides VI. A simple synthesis of N-acylglycines of the benzoyl- and cinnamyl-type. Bull. Soc. Chim. Belg. 87:791-797.

11. Gornall, A. G., C. J. Bardawill, and M. M. David. 1949. Determination of serum proteins by means of the biuret reaction. J. Biol. Chem. 177:751-766.

12. Doumas, B. T., W. A. Watson, and H. C. Briggs. 1971. Albumin standards and the measurement of serum albumin with bromcresol green. Clin. Chim Acta. 31:87-96.

13. Laurell, S., and G. Tibbling. 1967. Colorimetric micro-determination of free fatty acids in plasma. Clin. Chim. Acta. 16:57-62. 
14. Roth, M. 1967. Dosage fluorimétrique de la bilirubine. Clin. Chim. Acta. 17:487-492.

15. Lichtenwalner, D. M., B. Suh, B. Lorber, and A. M. Sugar. 1979. New rapid assay for nafcillin in serum by spectrofluorometry. Antimicrob. Agents Chemother. 16:210-213.

16. Singhvi, S. M., A. F. Heald, H. H. Gadebusch, M. E. Resnick, L. R. Difazio, and M. A. Leitz. 1977. Human serum protein binding of cephalosporin antibiotics in vitro. J. Lab. Clin. Med. 89:414-420.

17. Krasner, J., G. P. Giacoia, and S. J. Yaffe. 1973. Drug-protein binding in the newborn infant. Ann. NY Acad. Sci. 226:101-114.

18. Miyoshi, K., K. Saijo, Y. Kotani, T. Kashiwagi, and H. Kawai. 1966. Characteristic properties of fetal human albumin (Alb. F) in isomerization equilibrium. Tokushima J. Exp. Med. 13:121-132.

19. Wallace, S. 1977. Altered plasma albumin in the newborn infant. Br. J. Clin. Pharmacol. 4:82-85.

20. Odell, G. B. 1973. Influence of binding on the toxicity of bilirubin. Ann. NY Acad. Sci. 226:225-237.

21. Rane, A., P. K. M. Lunde, B. Jalling, S. J. Yaffe, and F. Sjöqvist. 1971. Plasma protein binding of diphenylhydantoin in normal and hyperbilirubinemic infants. J. Pediatr. 78:877-882.

22. Spector, A. A., E. C. Santos, J. D. Ashbrook, and J. E. Fletcher. 1973. Influence of free fatty acid concentration on drug binding to plasma albumin. Ann. NY Acad. Sci. 226:247-258.
23. Morselli, P. L., R. Franco-Morselli, and L. Bossi. 1983. Clinical pharmacokinetics in newborns and infants: age-related differences and therapeutic implications. In Handbook of Clinical Pharmacokinetics. M. Gibaldi and L. Prescott, editors. ADIS Health Science Press, New York. 98-141.

24. Suh, B., H. W. Lee, S. Y. Hong, S. Kim, J. Eshraghi, and W. K. Paik. 1986. A new HPLC analytical method for $o$-hydroxyhippuric acid in uremic serum. J. Biochem. Biophys. Methods. 13:211-220.

25. Levy, G. 1975. Salicylate pharmacokinetics in the human neonate. In Basic and Therapeutic Aspects of Perinatal Pharmacology. P. L. Morselli, S. Garattini, and F. Sereni, editors. Raven Press, New York. 319-330.

26. Gladtke, E., and G. Heimann. 1975. The rate of development of elimination functions in kidney and liver of young infants. In Basic and Therapeutic Aspects of Perinatal Pharmacology. P. L. Morselli, S. Garattini, and F. Sereni, editors. Raven Press, New York. 393-403.

27. Feingold, B. F. 1968. Recognition of food additives as a cause of symptoms of allergy. Ann. Allergy. 26:309-313.

28. Booth, A. N., D. J. Robbins, and W. L. Dunkley. 1962. Occurence of salicyluric acid in milk. Nature (Lond.). 194:290-291.

29. Speer, F. 1979. Allergy to methyl salicylate. Ann. Allergy. 43:36-37. 